\title{
Can the Human Brain Predict the Consequences of Arm Movement Corrections When Transporting an Object? Hints from Grip Force Adjustments
}

\author{
Frédéric Danion and Fabrice R. Sarlegna \\ Unité Mixte de Recherche Mouvement et Perception, Centre National de la Recherche Scientifique, Université de la Méditerranée, 13288 Marseille, France
}

It is well established that motor prediction is crucial for many of our daily actions. However, it is still unclear whether the brain generates motor prediction in real time. To challenge this idea, grip force was monitored while subjects had to transport a hand-held object to a visual target that could move unexpectedly. In agreement with previous reports, subjects triggered fast arm movement corrections to bring the object to the new target location. In addition, we found that subjects initiated grip force adjustments before or in synchrony with arm movement corrections. Throughout the movement, grip force anticipated the mechanical consequences resulting from arm motion, even when it was substantially corrected. Moreover, the predictive control of grip force did not interfere with the on-line control of arm trajectory. Altogether, our results suggest that motor prediction is an automatic, real-time process operating during movement execution and correction.

Key words: motor prediction; motor control; grip force; double-step paradigm; hand; human

\section{Introduction}

There is growing evidence that skilled motor behavior relies on the ability of the brain to predict the outcome of volitional acts (Desmurget and Grafton, 2000; Flanagan et al., 2006). Anticipatory mechanisms have been demonstrated in various motor tasks such as eye-hand coordination (Vercher et al., 2003) and object manipulation (Flanagan and Wing, 1997). For instance, when holding an object, it is well established that grip force (GF) is adjusted in advance with respect to the mechanical consequences of the arm movement, a control mode efficient to prevent the object to slip away (Johansson and Westling, 1988; Flanagan et al., 2003; Nowak et al., 2004; Danion et al., 2007). Current theories suggest that the human CNS performs predictions based on neural networks that capture the causal relationship between the generated motor commands and the resulting body motion (Wolpert and Flanagan, 2001). Such neural networks have been described as forward internal models (Kawato, 1999).

An implicit postulate of the forward-modeling theory is that the brain predicts movement outcome in real time (Desmurget and Grafton, 2000) by using a copy of the current motor commands (efference copy). However, to our knowledge, accurate motor prediction has only been shown for actions whose original plan remained unchanged throughout movement execution (Flanagan and Wing, 1997; Ariff et al., 2002; Descoins et al.,

Received July 9, 2007; revised Sept. 24, 2007; accepted 0ct. 16, 2007.

We thankF. Buloup and C. Bourdin for technical support, as well as V. Jirsa, P. M. Bernier, and the two anonymous reviewers for their insightful comments on previous drafts of this manuscript.

Correspondence should be addressed to Frédéric Danion, Unité Mixte de Recherche Mouvement et Perception, Faculté des Sciences du Sport, 13288 Marseille Cedex 09, France. E-mail: frederic.danion@univmed.fr.

D01:10.1523/JNEUROSCI.3110-07.2007

Copyright $\odot 2007$ Society for Neuroscience $\quad$ 0270-6474/07/2712839-05\$15.00/0
2006). Therefore, it is possible that motor prediction is defined before movement onset, as originally proposed for the motor commands underlying the movement (Keele, 1968). We reasoned that, if motor prediction is a real-time process, it should remain accurate even if humans change their motor commands during movement execution.

To get additional insight into the dynamics of motor prediction, we used the so-called "double-step paradigm," in which reaching arm movements are performed toward a target that jumps unexpectedly near movement onset (Georgopoulos et al., 1981; Goodale et al., 1986; Desmurget et al., 2004). This paradigm was selected because it elicits fast motor corrections that allow reaching the displaced target before movement offset (Day and Brown, 2001; Prablanc et al., 2003; Sarlegna et al., 2003). An original feature of the present study was that arm movements had to be performed while holding an object. The main objective was to determine whether grip force would remain adequately controlled with respect to changes in arm kinematics. We hypothesized that, if motor prediction is performed in real time, the anticipatory features of grip force control observed under natural (i.e., unperturbed) conditions should persist despite the initiation of fast motor corrections. Specifically, we expected grip force corrections to be functional and precede (or occur simultaneously with) movement corrections. Additionally, we assessed whether the anticipatory control of grip force interferes with arm movement corrective mechanisms by repeating the task without holding the object.

\section{Materials and Methods}

Subjects. Eight self-proclaimed right-handed males (29.2 \pm 4.3 years old; data present mean \pm interindividual SD throughout the manuscript) 
participated in the experiment. All were healthy and gave informed consent according to university regulations.

Apparatus. The hand-held object was a force sensor (ELPM-T1M-50N; Entran, Fairfield, $\mathrm{NJ}$ ) measuring the force perpendicular to its surface (i.e., GF) exerted by the thumb and index finger (Fig. 1). The object, covered with sandpaper, was attached to an elastic cord (stiffness, $38.3 \mathrm{~N} / \mathrm{m}$ ) such that the minimum grip force to prevent slip of the object increased linearly as a function of movement amplitude, i.e., load force (LF). Across subjects, the friction coefficient was estimated at $1.12 \pm 0.14$. The object was equipped with an accelerometer $\left( \pm 20 \mathrm{~m} / \mathrm{s}^{2}\right.$; EGCS-FS-2, Entran). An infraredsensitive camera (C2399; Hamamatsu, Shizuoka, Japan) tracking an infrared-emitting source mounted on the object measured its horizontal displacement. Overall, the object weighted $0.045 \mathrm{~kg}$. Another force sensor (ELPM-T1M-25N; Entran) was attached to the elastic cord to determine LF when displacing the object. All signals were collected at $1000 \mathrm{~Hz}$.

Procedure. Seated subjects had to displace with their right-hand the instrumented object to a visual target along the frontoparallel plane. Before each trial, subjects placed their hand at a start location prescribed by a light-emitting diode (LED). At start position, the elastic load was $\sim 10 \mathrm{~N}$. After $1 \mathrm{~s}$, the start LED was extinguished while a target LED was turned on. Subjects were asked to first look at the target and then bring the object to the target. The target was 8,16 , or $24 \mathrm{~cm}$ away from the starting position (corresponding to an elastic load of 13, 16 , and $19 \mathrm{~N}$, respectively). In some trials for which the middle target was illuminated, the middle target was extinguished when movement speed exceeded $9 \mathrm{~cm} / \mathrm{s}$ while the near or far target appeared. On average, targets jumped $26 \pm 7 \mathrm{~ms}$ after movement onset. Subjects were informed to reach the final target location and remain stationary until the target disappeared (i.e., $3 \mathrm{~s}$ after the starting LED extinction). Altogether, subjects could face five experimental conditions: three toward stationary targets $(8$, 16 , and $24 \mathrm{~cm})$ and two with target displacements $(16 \rightarrow 8$ and $16 \rightarrow 24 \mathrm{~cm})$. Randomization of the conditions ensured that final target location was unpredictable. Each participant completed 50 trials (10 trials per condition), preceded by 10 practice trials (two trials per condition), per session.

To investigate the possible impact of predictive grip force control on arm movement corrective mechanisms, all subjects performed two sessions. Subjects transported the object by either holding it between the thumb and the index finger ("grasp") or simply moving the index fingertip onto which the object was securely attached ("no grasp"). In the grasp session, subjects received verbal feedback before movement initiation to start approximately with the same initial GF (mean of $16.3 \pm 0.3 \mathrm{~N}$ ). Session order was counterbalanced across subjects.

Data analysis. Kinematic and kinetic signals were low-pass filtered at $10 \mathrm{~Hz}$ (fourth-order, no-lag, dual-pass Butterworth). Because of the reduced mass of the object, the contribution of the inertial load $(<0.25 \mathrm{~N})$ to total load force $(>10 \mathrm{~N})$ was small. Movement onset and offset were computed using the derivative of the positional signal with a threshold of $4 \mathrm{~cm} / \mathrm{s}$. Similar movement onsets were obtained using the LF derivative (threshold of $2 \mathrm{~N} / \mathrm{s}$ ). GF onset was assessed using a $2 \mathrm{~N} / \mathrm{s}$ threshold (Danion, 2007).

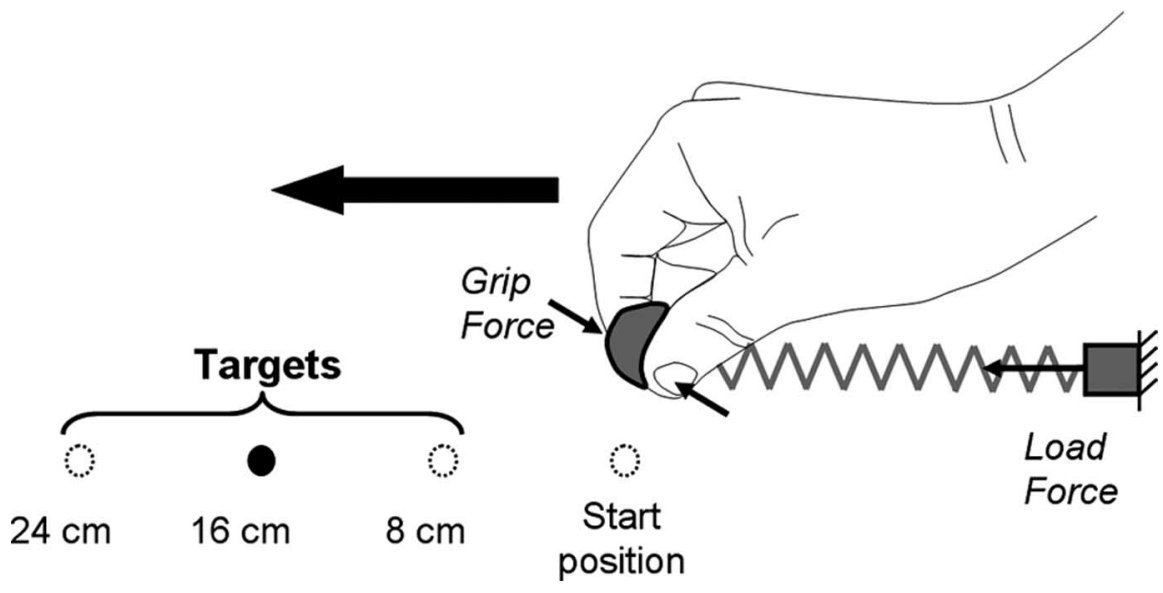

Figure 1. Experimental setup in the grasp session.
A
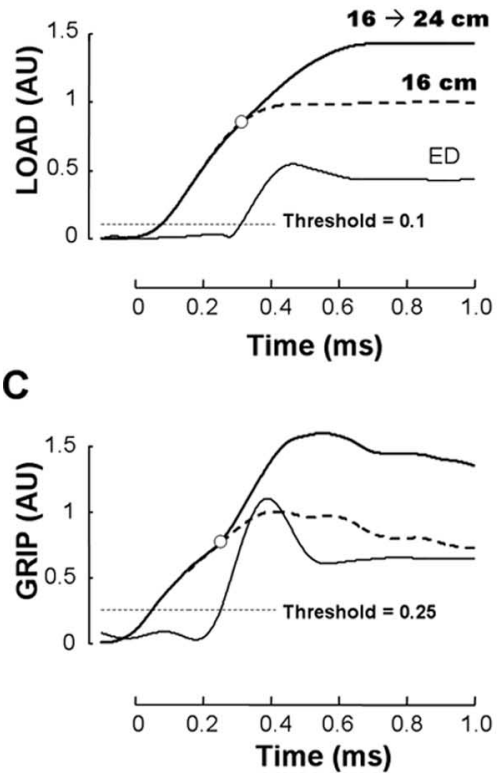

B

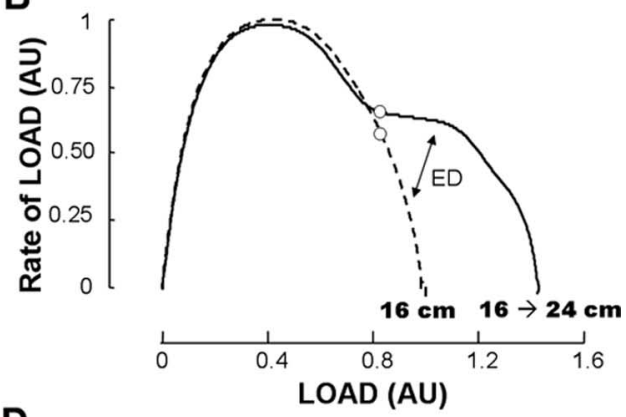

D

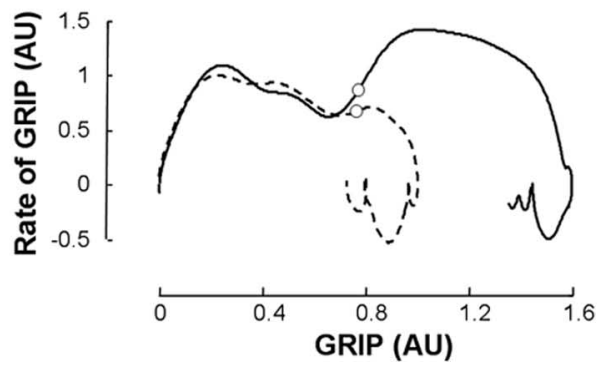

Figure 2. Average load and grip force trajectories during the 16 and $16 \rightarrow 24 \mathrm{~cm}$ conditions for a representative subject in the grasp session. $\boldsymbol{A}$ and $\boldsymbol{C}$ present normalized force as a function of time. $\boldsymbol{B}$ and $\boldsymbol{D}$ present normalized phase portraits of force trajectories (force rate vs force). White circles show when the force trajectories start to differ significantly. AU, Arbitrary units.

To determine the latencies of arm movement and grip force corrections in double-step conditions, a single procedure was developed. GF and LF trajectories after movement onset were averaged for each subject in each experimental condition. Then, we drew normalized twodimensional phase portraits of GF and LF (Fig. 2B,D). Amplitude normalization required that, for each subject, peak force and peak force rate both reached a value of 1 in the $16 \mathrm{~cm}$ condition (this condition serving as reference for the other conditions). To determine when force trajectories in $16 \rightarrow 8$ and $16 \rightarrow 24 \mathrm{~cm}$ conditions diverged significantly from that in the $16 \mathrm{~cm}$ condition, the Euclidian distance (ED) between pairs of trajectories was computed separately for GF and LF at each instant of time $(t)$ within the normalized phase space (Jirsa and Kelso, 2005). ED takes into account both force $(F)$ and force rate $(d F)$ and is defined as follows: $\operatorname{ED}^{2}(t)=\left[F_{1}(t)-F_{2}(t)\right]^{2}+\left[d F_{1}(t)-d F_{2}(t)\right]^{2}$.

Force corrections were determined when ED exceeded a threshold set for all subjects to 0.1 for LF (Fig. $2 A$ ) and 0.25 for GF (Fig. 2C). A higher threshold was chosen for GF compared with LF because of larger background fluctuations, but note that having a higher threshold for GF reflects a conservative choice with respect to our hypothesis because it is 
likely to delay GF corrections compared with LF corrections. This procedure yielded, for each subject, a mean time of first corrections of GF and LF for each double-step condition.

To further investigate the temporal relationship between GF and LF, we developed a procedure to estimate the current lag between both signals (see Fig. 3). For each subject and each condition, average force trajectories were normalized in amplitude so that forces at movement onset and offset were, respectively, 0 and 1 . Then we determined, at each instant of time, the minimal delay such that LF value matched GF value.

ANOVAs [2 (grasp, no grasp) $\times 5$ experimental conditions] and Newman-Keuls post hoc analyses were used to test the effect of our experimental factors, with a 0.05 significance criterion. Because correlation coefficients do not follow a normal distribution, logarithmic transformations ( $z$-score) were used before conducting statistical procedures.

\section{Results}

\section{Arm movement kinematics}

Despite the unexpected target jumps, subjects were able to reach the final target location before movement offset (mean error $<4$ $\mathrm{mm}$ in all experimental conditions). Movements lasted $472 \mathrm{~ms}$ in the $16 \mathrm{~cm}$ stationary target and were longest when the target was displaced ( $p<0.001$ ), reaching 770 and $696 \mathrm{~ms}$ on average for the $16 \rightarrow 8$ and $16 \rightarrow 24 \mathrm{~cm}$ conditions, respectively. Holding the object did not influence significantly movement time (mean of $575 \mathrm{~ms}$ ), peak velocity (mean of $0.62 \mathrm{~m} / \mathrm{s}$ ), peak acceleration (mean of $5.4 \mathrm{~m} / \mathrm{s}^{2}$ ), and their associated latencies (mean of 210 and $83 \mathrm{~ms}$, respectively). Altogether, arm movement kinematics were similar in grasp and no grasp sessions, as supported by high correlation coefficients between LF signals $(R>0.99)$.

Figure $2 A$ presents LF trajectories for the 16 and $16 \rightarrow 24 \mathrm{~cm}$ conditions, with the Euclidian distance between the two trajectories to determine when they started to differ. In this example, ED exceeded the threshold $307 \mathrm{~ms}$ after movement onset. Because the target jumped $27 \mathrm{~ms}$ after movement initiation for this subject, we considered $280 \mathrm{~ms}$ as the latency of first movement correction. This procedure yielded an average latency of $284 \pm 18 \mathrm{~ms}$ for arm movement corrections. The ANOVA revealed that neither the direction of target jump nor the grasp/no grasp factor significantly influenced this latency, as illustrated in Figure 3.

\section{Grip force corrections}

In perturbed trials, grip force was modified such that its final value, averaged over the $100 \mathrm{~ms}$ after movement offset, was adequate with respect to the final hand location. Indeed, there was no significant difference between final GF in 8 and $16 \rightarrow 8 \mathrm{~cm}$ conditions (mean of 21.2 and $21.9 \mathrm{~N}$, respectively), as well as between 24 and $16 \rightarrow 24 \mathrm{~cm}$ conditions (mean of 32.6 and $32.3 \mathrm{~N}$, respectively). Figure $2 C$ shows average GF trajectories from a representative subject. Here, we found that first grip force corrections occurred, on average, $200 \mathrm{~ms}$ after the target displacement for this subject. Overall, we found that the latency of GF corrections was $209 \pm 54 \mathrm{~ms}$ after $16 \rightarrow 24 \mathrm{~cm}$ target jumps. When the target jumped backward, Figure 3 indicates that the latency was longer ( $346 \pm 160 \mathrm{~ms} ; p=0.03$ ), presumably because the associated arm movement corrections did not threaten the grip force safety margin.

An important issue was to determine whether grip force corrections preceded or followed arm movement corrections. The ANOVA revealed that, after $16 \rightarrow 24 \mathrm{~cm}$ target jumps, GF latency was smaller than LF latency $(p<0.001)$. This anticipatory behavior was observed in all subjects: on average, GF corrections preceded LF corrections by $71 \pm 24 \mathrm{~ms}$. This temporal difference was not significantly correlated with the latency of movement corrections $(R=-0.48 ; p>0.05)$. When the target jumped backward, GF corrections occurred simultaneously with LF cor-

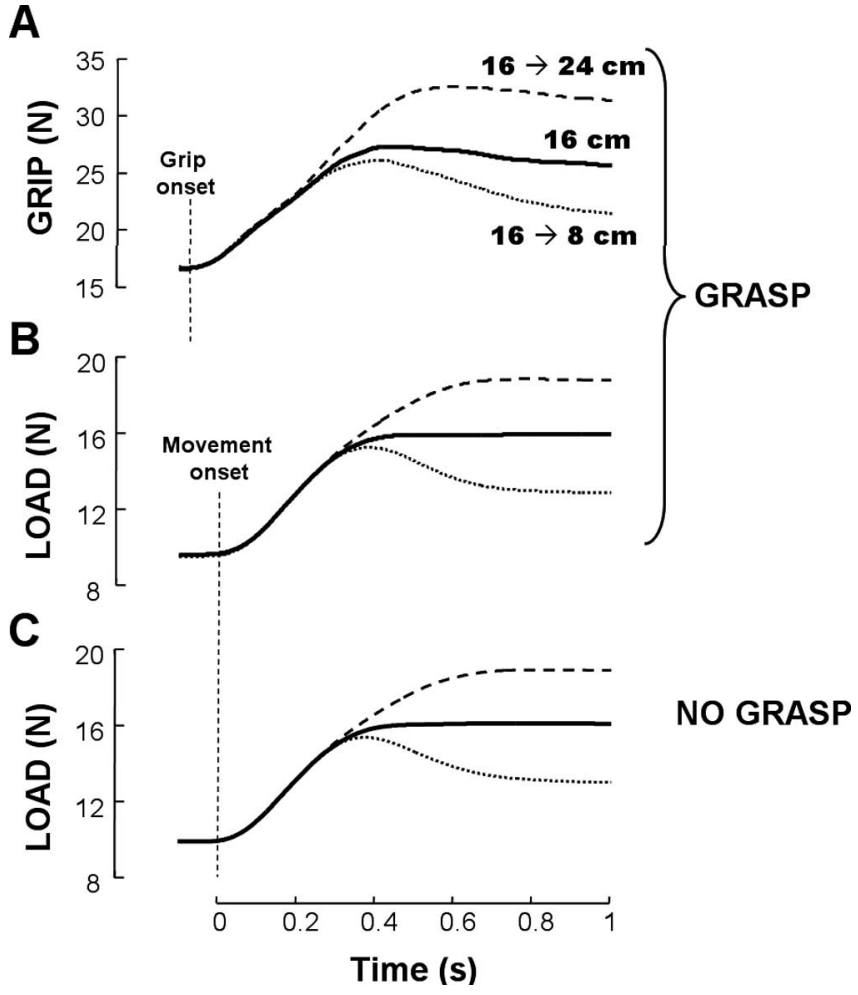

Figure 3. Ensemble average grip and load force profiles during unperturbed ( $16 \mathrm{~cm}$ only) and perturbed $(16 \rightarrow 24$ and $16 \rightarrow 8 \mathrm{~cm}$ ) trials in grasp and no grasp sessions.

rections, as suggested by the nonsignificant difference between GF and LF latencies.

\section{Grip-load force coordination}

Grip-load force coupling was high in all experimental conditions as revealed by average correlation coefficients $(R>0.91)$. A main effect of condition was observed $(p<0.001)$, and post hoc analyses revealed smaller $R$ values for the 8 and $16 \rightarrow 8 \mathrm{~cm}$ conditions (mean of $0.92 \pm 0.06$ ) compared with the other conditions (mean of $0.98 \pm$ 0.02 ). Concerning the temporal relationship between GF and LF, we observed that GF was adjusted in a predictive, rather than reactive, manner. Indeed, GF increase preceded LF increase (i.e., arm movement onset) by $71 \pm 11 \mathrm{~ms}$ on average (Fig. 3); this delay did not differ significantly between perturbed and unperturbed conditions. The lag between GF and LF was further examined around the time of first grip force corrections (from -100 to $+100 \mathrm{~ms}$, by $25 \mathrm{~ms}$ steps). For comparison purposes, this lag was evaluated in the $16 \mathrm{~cm}$ condition over the corresponding time windows. ANOVAs revealed no significant effects of target jump or time step. On average, grip force preceded load force by $22 \pm 18 \mathrm{~ms}$. Therefore, subjects did not wait for an increase in the tension of the elastic cord to increase their grip force but anticipated, throughout movement execution (Fig. 4), the consequences of their arm movements on the load force at the object-finger interface. In summary, we found no direct evidence that target jumps influenced the coordination between GF and LF.

\section{Discussion}

In the present experiment, we monitored grip force during goaldirected arm movements to estimate whether the human brain has the ability to anticipate in real time the consequences of movement corrections. Our main finding is that the grip-load force coupling is preserved despite the initiation of fast arm movement corrections in response to unexpected target jumps. 
A

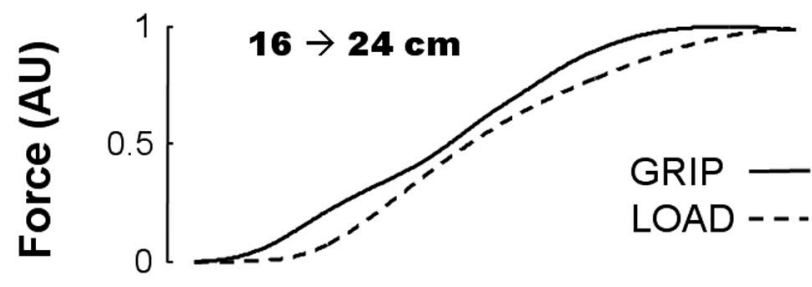

B

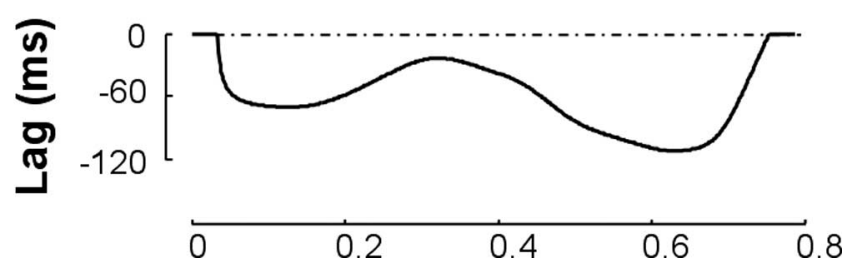

Figure 4. Grip force and load force coordination during a $16 \rightarrow 24 \mathrm{~cm}$ target jump in the grasp session (averaged signals for a representative subject). $\boldsymbol{A}$, Temporal evolution of normalized grip and load force trajectories. $\boldsymbol{B}$, Temporal evolution of the lag between grip and load force. A negative value of the lag indicates that grip force precedes load force. Note that this is actually the case throughout movement execution. AU, Arbitrary units.

Specifically, grip force adjustments were tightly coupled to arm movement corrections at both the spatial and temporal level. We even noticed that grip force corrections could precede arm movement corrections. We also observed that holding the object or not did not influence the latency of arm movement corrections. Assuming that grip force is driven by predictive mechanisms under nonperturbed conditions (Flanagan and Wing, 1997; Kawato, 1999; Wolpert and Flanagan, 2001), our results suggest that those predictive mechanisms can be described as real-time, low-demanding processes continuously engaged during movement execution.

Do grip force corrections reflect a predictive mechanism? In the present study, a default, reflex-like response could have been to increase grip force to secure the object in the hand independently of the intended arm movement correction. In contrast, grip force adjustments were strongly determined by the direction of the target jump. Indeed, when the target jumped forward, grip force increased, whereas it decreased when the target jumped backward. However, it is worth noting that, compared with backward target jumps, grip force corrections after forward jumps were initiated earlier while preserving higher grip-load force coupling. This context-dependent effect is consistent with studies showing that subjects adjust their grip force more efficiently and rapidly when reacting to unexpected increasing loads compared with decreasing loads (Johansson and Westling, 1988; Mrotek et al., 2004).

In addition to a spatial coupling, we observed a tight temporal coupling with grip force corrections preceding arm motor corrections. We interpret this finding as evidence that grip force corrections are controlled in a feedforward manner. Among the possible feedforward mechanisms, a first option evoked by $\mathrm{Ka}$ wato et al. (2003) is that arm movement and grip force are driven by a unique controller, as also evoked by Slijper et al. (2002) for arm movement and anticipatory postural adjustments. Along this scheme, grip force and movement corrections would be con- sidered as elements of the same single action and, earlier responses in the fingers could be facilitated by faster neural motor pathways and/or lower inertia. This possibility is supported by studies showing that the fast corticospinal tract contributes more to the control of distal muscles than proximal muscles (Turton and Lemon, 1999). However, it becomes unclear why, in a reachand-grasp task, grip aperture corrections follow arm movement corrections when object location or size changes unexpectedly (Paulignan and Jeannerod, 1996). Moreover, the fact that grip force still precedes load force when pulling/pushing a static load (De Freitas et al., 2007) suggests that inertia is not fully responsible for the anticipatory nature of grip force adjustments. Last but not least, Kawato et al. (2003) suggested that, if humans had a unique controller for arm and finger muscles, the rate of learning for grip force and arm movement should be identical when manipulating novel objects, but Flanagan et al. (2003) found different learning rates.

An alternative option is to consider that grip force is adjusted by predictive mechanisms that anticipate movement outcome (Flanagan and Wing, 1997; Wolpert and Flanagan, 2001). Currently, we favor this forward-modeling approach because it accounts for the fact that grip force corrections preceded arm movement corrections. Second, it justifies why grip force adjustments were functionally tuned by the changes in arm motion. Third, it is consistent with the experimental evidence of separate controllers for arm movement and grip force (Flanagan et al., 2003). However, accepting that motor prediction underlies grip force adjustments implies that the efference copy, a key component, must remain fully accessible to the predictor when movement corrections are generated (Kawato, 1999; Sommer and Wurtz, 2002). This assertion is not trivial because distinct neural pathways could underlie corrected and uncorrected movements (Pisella et al., 2000; Day and Brown, 2001; Desmurget et al., 2004).

\section{Is grip force controlled in real time during object manipulation?}

Efficient motor prediction has been widely demonstrated when the intended movement unfolds smoothly (Flanagan and Wing, 1997; Nowak et al., 2004; Danion et al., 2007). Previous work on eye-hand coordination has consistently shown that unexpected events such as mechanical perturbations lead to transitory deficits in terms of motor prediction of the upper-limb motion (Ariff et al., 2002; Vercher et al., 2003). Mechanical perturbations alter limb dynamics and require the modification of sensorimotor transformations, presumably by updating both inverse and forward internal models (Shadmehr and Mussa-Ivaldi, 1994; Wolpert and Flanagan, 2001). In contrast, the visual perturbation used in the present study led to a change in movement kinematics originating purely from a change in motor commands. Within this simpler context, we showed that grip force can be adjusted on-line according to the correction of rapid arm movements. Based on the assumption that grip force is driven by predictive mechanisms, we suggest that the human brain is able to generate motor prediction rapidly and accurately when the intended movement is corrected in response to a target jump. We conclude that, when limb dynamics are not altered, motor prediction appears as a real-time process that is operating throughout movement execution.

This conclusion fits well with a seminal study of Johansson and Westling (1988) who investigated grip force control when lifting an object heavier than expected. Although not explicitly measured, the grip-load force coupling seemed preserved despite the initiation of movement corrections (Johansson and Westling, 1988, their Fig. 4). Our conclusion is also consistent with a study suggesting that, when standing subjects reach for displaced tar- 
gets, postural adjustments counteract the effect of hand trajectory corrections (Martin et al., 2003). However, our results seem to conflict with data showing a slight alteration of the grip-load force coupling when subjects have to initiate arm movements as quickly as possible after the appearance of a target (Flanagan and Lolley, 2001). We believe that part of this discrepancy lies in the fact that, in our study, movements were restricted along a straight line with an elastic, position-dependant load, whereas in the study of Flanagan and Lolley, movements could be performed in any direction with an inertial, acceleration-dependent load (Danion et al., 2007). Altogether, this could make our task somewhat simpler from a computational perspective.

\section{Is predictive grip force control a demanding process?}

To assess whether anticipatory grip force control is a computationally demanding process, arm movements were performed with and without holding the object. In both situations, the large, consciously perceived target displacements resulted in arm movement corrections initiated with a similar latency. This latency, within the range of values reported previously (Georgopoulos et al., 1981; Sarlegna et al., 2003), presumably reflects voluntary rather than automatic modifications of the initial motor plan (Day and Lyon, 2000; Desmurget et al., 2004; Johnson and Haggard, 2005). We interpret the lack of grasp/no grasp difference as possible evidence that the predictive mechanisms engaged in the control of grip force are low-demanding processes. Recent theories of motor control suggest that motor prediction is a neural process that is engaged in arm movement production even when we do not hold an object or track our hand with the eye. The main advantage of this scheme is to predict sensory events (Wolpert and Flanagan, 2001) and allow internal feedback strategies to control fast-reaching movements (Desmurget and Grafton, 2000). Hypothesizing that the brain constantly predicts the outcome of arm movements, it may appear trivial that arm movement corrections were not retarded by holding the object. However, although moving a limb and transporting an object both rely on the ability to anticipate the future state of the limb, avoiding the slip of the object requires the additional ability to anticipate the mechanical consequences of the limb state on the load force at the object-finger interface (Descoins et al., 2006). The present study indicates that predicting the movement resulting load is a low-demanding process because it does not interfere with the ability to perform fast motor corrections. The view that motor prediction could be an automatic and low-demanding process is reminiscent of the view provided by other researchers for the process of motor correction (Goodale et al., 1986; Day and Lyon, 2000; Pisella et al., 2000; Prablanc et al., 2003).

\section{References}

Ariff G, Donchin O, Nanayakkara T, Shadmehr R (2002) A real-time state predictor in motor control: study of saccadic eye movements during unseen reaching movements. J Neurosci 22:7721-7729.

Danion F (2007) The contribution of non-digital afferent signals to grip force adjustments evoked by brisk unloading of the arm or the held object. Clin Neurophysiol 118:146-154.

Danion F, Descoins M, Bootsma R (2007) Aging affects the predictive control of grip force during object manipulation. Exp Brain Res 180:123-137.

Day BL, Brown P (2001) Evidence for subcortical involvement in the visual control of human reaching. Brain 124:1832-1840.

Day BL, Lyon IN (2000) Voluntary modification of automatic arm movements evoked by motion of a visual target. Exp Brain Res 130:159-168.

De Freitas Jr PB, Krishnan V, Jaric S (2007) Elaborate force coordination of precision grip could be generalized to bimanual grasping techniques. Neurosci Lett 412:179-184.

Descoins M, Danion F, Bootsma R (2006) Predictive control of grip force when moving an object with an elastic load applied on the arm. Exp Brain Res 172:331-342.
Desmurget M, Grafton S (2000) Forward modelling allows feedback control for fast reaching movements. Trends Cogn Sci 4:423-431.

Desmurget M, Gaveau V, Vindras P, Turner RS, Broussolle E, Thobois S (2004) On-line motor control in patients with Parkinson's disease. Brain 127:1755-1773.

Flanagan JR, Lolley S (2001) The inertial anisotropy of the arm is accurately predicted during movement planning. J Neurosci 21:1361-1369.

Flanagan JR, Wing AM (1997) The role of internal models in motion planning and control: evidence from grip force adjustments during movements of hand-held loads. J Neurosci 17:1519-1528.

Flanagan JR, Vetter P, Johansson RS, Wolpert DM (2003) Prediction precedes control in motor learning. Curr Biol 13:146-150.

Flanagan JR, Bowman MC, Johansson RS (2006) Control strategies in object manipulation tasks. Curr Opin Neurobiol 16:650-659.

Georgopoulos AP, Kalaska JF, Massey JT (1981) Spatial trajectories and reaction times of aimed movements: effects of practice, uncertainty, and change in target location. J Neurophysiol 46:725-743.

Goodale MA, Pelisson D, Prablanc C (1986) Large adjustments in visually guided reaching do not depend on vision of the hand or perception of target displacement. Nature 6064:748-750.

Jirsa VK, Kelso JAS (2005) The excitator as a minimal model for the coordination dynamics of discrete and rhythmic movement generation. J Mot Behav 37:35-51.

Johansson RS, Westling G (1988) Coordinated isometric muscle commands adequately and erroneously programmed for the weight during lifting task with precision grip. Exp Brain Res 71:59-71.

Johnson H, Haggard P (2005) Motor awareness without perceptual awareness. Neuropsychologia 43:227-237.

Kawato M (1999) Internal models for motor control and trajectory planning. Curr Opin Neurobiol 9:718-727.

Kawato M, Kuroda T, Imamizu H, Nakano E, Miyauchi S, Yoshioka T (2003) Internal forward models in the cerebellum: fMRI study on grip force and load force coupling. Prog Brain Res 142:171-188.

Keele SW (1968) Movement control in skilled motor performance. Psychol Bull 70:387-403.

Martin O, Julian B, Boissieux L, Gascuel JD, Prablanc C (2003) Evaluating online control of goal-directed arm movement while standing in a visual environment. J Visual Comput Anim 14:253-260.

Mrotek LA, Hart BA, Schot PK, Fennigkoh L (2004) Grip responses to object load perturbations are stimulus and phase sensitive. Exp Brain Res 155:413-420.

Nowak DA, Hermsdorfer J, Rost K, Timmann D, Topka H (2004) Predictive and reactive finger force control during catching in cerebellar degeneration. Cerebellum 3:227-235.

Paulignan Y, Jeannerod M (1996) Prehension movements: the visuomotor channels hypothesis revisited. In: Hand and brain: the neurophysiology and psychology of hand movements (Wing AM, Haggard P, Flanagan JR, eds), pp 265-282. San Diego: Academic.

Pisella L, Grea H, Tilikete C, Vighetto A, Desmurget M, Rode G, Boisson D, Rossetti (2000) An "automatic pilot" for the hand in human posterior parietal cortex: toward reinterpreting optic ataxia. Nat Neurosci 3:729-736.

Prablanc C, Desmurget M, Gréa H (2003) Neural control of on-line guidance of hand reaching movements. Prog Brain Res 142:155-170.

Sarlegna F, Blouin J, Bresciani JP, Bourdin C, Vercher JL, Gauthier GM (2003) Target and hand position information in the online control of goal-directed arm movements. Exp Brain Res 151:524-535.

Shadmehr R, Mussa-Ivaldi FA (1994) Adaptive representation of dynamics during learning of a motor task. J Neurosci 14:3208-3224.

Slijper H, Latash ML, Mordkoff JT (2002) Anticipatory postural adjustments under simple and choice reaction time conditions. Brain Res 924:184-197.

Sommer MA, Wurtz RH (2002) A pathway in primate brain for internal monitoring of movements. Science 296:1480-1482.

Turton A, Lemon RN (1999) The contribution of fast corticospinal input to the voluntary activation of proximal muscles in normal subjects and in stroke patients. Exp Brain Res 129:559-572.

Vercher JL, Sares F, Blouin J, Bourdin C, Gauthier GM (2003) Role of sensory information in updating internal models of the effector during arm tracking. Prog Brain Res 142:203-222.

Wolpert DM, Flanagan JR (2001) Motor prediction. Curr Biol 11:R729_ R732. 